
\title{
28 Research Suare \\ Plasma thiol redox status as indicator of acute kidney injury
}

\section{Lisanne Boekhoud ( $\square$ l.boekhoud@umcg.nl)}

Rijksuniversiteit Groningen

Jacqueline Koeze

Rijksuniversiteit Groningen

Elisabeth C. van der Slikke

Rijksuniversiteit Groningen

Arno R. Bourgonje

Rijksuniversiteit Groningen

Jill Moser

Rijksuniversiteit Groningen

Jan G Zijlstra

Rijksuniversiteit Groningen

Anneke C. Muller Kobold

Rijksuniversiteit Groningen

Marian L.C. Bulthuis

Rijksuniversiteit Groningen

Matijs van Meurs

Rijksuniversiteit Groningen

Harry van Goor

Rijksuniversiteit Groningen

Hjalmar R. Bouma

Rijksuniversiteit Groningen

\section{Research}

Keywords: sepsis, acute kidney injury (AKI), reactive oxygen species (ROS), oxidative stress, thiols, hydrogen sulphide.

Posted Date: August 5th, 2020

DOI: https://doi.org/10.21203/rs.3.rs-52235/v1

License: (9) (1) This work is licensed under a Creative Commons Attribution 4.0 International License. Read Full License 
Page $2 / 18$ 


\section{Abstract}

Introduction: Acute kidney injury (AKI) is associated with the abrupt loss of kidney function resulting in increased morbidity and mortality. Oxidative stress plays an important role in the pathophysiology of AKI. Free thiols (R-SH, sulfhydryl groups) are crucial components of the extracellular antioxidant machinery and reliably reflect systemic oxidative stress. Lower levels of thiols represent higher levels of oxidative stress. In this study, we hypothesized that plasma free thiols are associated with AKI upon admission to the intensive care unit (ICU).

Methods: In this study, 301 critically ill patients were included for analysis. Plasma samples were taken upon admission. Plasma levels of albumin-adjusted plasma free thiols were determined and correlated with AKI stage upon ICU admission.

Results: Albumin-adjusted plasma free thiols were significantly lower in patients with $\operatorname{AKI}(n=43$, median [interquartile range] $7.28 \mu \mathrm{mol} / \mathrm{g}[3.52,8.95])$ compared to patients without $\mathrm{AKI}(8.50 \mu \mathrm{mol} / \mathrm{g}[5.82,11.28]$; $p<0.05)$ upon admission to the ICU. Higher age $(B=-0.72, p<0.001)$, higher levels of neutrophil gelatinase-associated lipocalin $(B=-0.002, p<0.05)$, creatinine $(B=-0.01, p<0.05)$ and lower serum albumin $(B=0.47, p<0.001)$ were associated with lower free thiol levels. Further, albumin-adjusted free thiol levels were significantly reduced in patients with sepsis $(8.30[5.52-10.64] \mu \mathrm{mol} / \mathrm{g})$ compared to patients without sepsis $(6.95[3.72-8.92] \mu \mathrm{mol} / \mathrm{g} ; p<0.05)$.

Conclusion: Albumin-adjusted plasma free thiols were significantly reduced in patients with AKI and patients with sepsis compared with patients without AKI and sepsis. Together, these data suggest that free thiol levels are mainly reduced in sepsis-associated AKI.

\section{Introduction}

Acute kidney injury (AKI) is associated with increased morbidity and mortality, both at short and long-term (1). AKI is defined according the Kidney Disease: Improving Global Outcomes (KDIGO) guideline as an acute increase in serum creatinine and/or a decrease in urine output (2). AKI occurs in approximately 10$15 \%$ of hospitalized patients, while its incidence in intensive care units (ICUs) has been reported to exceed $50 \%$ (1). Although AKI is usually not the primary reason for ICU admission, it often complicates the clinical course of critically ill patients, and is associated with an increased risk of developing end-stage renal disease (ESRD), and mortality during and after hospitalization (3). In clinical practice, there is a lack of standardized preventive measures against AKI in critically ill patients (3). The most common cause of AKI in critically ill patients is sepsis (4), which is a life-threatening dysregulated host response to infection, leading to organ dysfunction. Sepsis has a relatively high incidence, with 48.9 million cases every year worldwide (5), and is associated with a poor prognosis. As such, one in three patients with sepsis will decease during hospital stay making it the leading cause of death among patients admitted to the ICU (6). The pathophysiology of sepsis-associated AKI consist of multiple factors, such as decreased renal blood flow, increased renal vascular resistance, endothelial dysfunction, infiltration of inflammatory 
cells in the renal parenchyma and obstruction of tubules with necrotic cells $(7,8)$. AKI is characterized by a complex pathophysiology; in which, amongst others, oxidative stress plays an important role (9).

Oxidative stress is defined as an imbalance between the production of reactive oxygen species (ROS) and a decreased availability of antioxidants (3). Sepsis is associated with an increased production of ROS (10). Activated immune system components and dysfunctional mitochondria play an important role in the generation of ROS in sepsis $(11,12)$. Overproduction of ROS leads to oxidative damage to mitochondria, DNA, lipids, and enzymes in the renal parenchyma (10), which together compromise renal function (3). Counteracting oxidative stress could be a potential strategy to prevent or treat AKI (5). In an ICU population, substitution of the recommended daily allowance of antioxidants as vitamin $\mathrm{C}$ improved the antioxidant capacity (13) and patients with sepsis who received antioxidants had a lower risk on developing AKI (14).

Measuring critical components of redox signalling could be a possible strategy to identify patients with higher levels of oxidative stress. Thiols are central components of the extracellular non-enzymatic antioxidant machinery (15). A thiol (R-SH, sulfhydryl group) is an organosulfur compound, that can scavenge free radicals (15). A reduction in free thiol groups reflects systemic oxidative stress since they are prime substrates for reactive species. Thiols are a robust and powerful biomarker for an individual's systemic reduction-oxidation (redox) status and are representative of the degree of systemic oxidative stress (16). High levels of systemic free thiols, as potent antioxidant substances, are reflective of a more favourable in vivo redox status (17). Plasma proteins, mainly albumin, contain the largest amount of redox-active thiol groups (approximately $75 \%$ of the total thiol pool) (18).

Since oxidative stress plays an important role in the aetiology of AKI in critically ill patients, thiols could potentially represent a biomarker to identify patients at risk of developing or progressing in AKI. In contrast to previous studies, that primarily focused on free thiols as disease biomarker, we will focus on thiols as a pathophysiological indicator. In this study, we therefore investigated whether thiols are associated with AKI in patients admitted to the ICU with and without sepsis. Based on the hypothesis that inflammation in sepsis augments oxidative stress (19), we also aimed to investigate associations between plasma free thiol levels and inflammatory biomarkers like C-reactive protein (CRP), calprotectin and neutrophil gelatinase-associated lipocalin (NGAL) (11).

\section{Material And Methods}

\section{Patient population, data collection and definitions}

Patients who were admitted to the intensive care unit (ICU) at the University Medical Center Groningen (UMCG) between January 2014 and April 2014 were included in this case-control study. Clinical data and blood samples were collected daily upon admission to the ICU until discharge or when patients had a prolonged stay, data was collected until day eight. The Medical Ethics Review Committee (in Dutch: 'Medische Ethische Toetsingscommissie', (METc)) of the UMCG reviewed and waived this study (METC 2013/174). Initially, 361 patients were included in the database. Patients of whom plasma samples were 
not available at day one of ICU admission were excluded $(n=60)$. Patients were stratified into groups based on the presence of AKI upon admission, defined by the KDIGO AKI score (Supplementary Table S1; Appendix)(2) based on the change in serum creatinine upon admission to the ICU as compared to the preexistent value in the year preceding ICU admission. In case no pre-existent creatinine value was available, the baseline creatinine was estimated using the Modification of Diet in Renal Disease (MDRD) based estimation method, assuming a creatinine clearance of $75 \mathrm{ml} / \mathrm{min} / 1.73 \mathrm{~m}^{2}(20)$. Besides AKI stage, continuous change in serum creatinine was used and calculated as change in pre-existent serum creatinine and serum creatinine upon admission. After the first day of admission, when diuresis was known, AKI was defined based on the KDIGO criteria using both serum creatinine and diuresis (Supplementary Table S1; Appendix). AKI progression was defined as any increase in KDIGO stage within 48 hours. Presence of sepsis was determined based on the sequential organ failure assessment (SOFA) score (21). Sepsis was defined as two or more points in the SOFA criteria and microbiological evidence for an infection.

\section{Measurement of plasma free thiol levels}

Plasma free thiol concentrations were measured as previously described, with minor modifications $(22,23)$. Plasma samples were stored at $-80^{\circ} \mathrm{C}$ until analysis of free thiol levels. First, samples were thawed on ice overnight, followed by centrifugation at $10,000 \mathrm{rpm}$ for 10 minutes at $4^{\circ} \mathrm{C}$. A calibration curve with L-cysteine (Fluka Biochemika, Buchs, Switzerland) standard curve [15.625 $\mu \mathrm{M}$ to $1000 \mu \mathrm{M}]$ was made in the same $0.1 \mathrm{M}$ Tris/EDTA buffer ( $\mathrm{pH} 8.2$ ). Next, $75 \mu \mathrm{L}$ plasma was 4 -fold diluted with $0.1 \mathrm{M}$ Tris/EDTA buffer ( $\mathrm{pH} 8.2$ ) and added to a flat bottom 96-well plate in triplicates. After 20 minutes of incubation at room temperature, the absorbance was measured at $630 \mathrm{~nm}$, while absorbance at $412 \mathrm{~nm}$ was subtracted as background. Next, $20 \mu \mathrm{L} 1.9 \mathrm{mM}$ DTNB (5,5'-dithio-bis (2-nitrobenzoic acid) Ellman's Reagent, Sigma Aldrich Corporation, St. Louis, MO, USA) in phosphate buffer $(0.1 \mathrm{M}, \mathrm{pH} 7)$ was added followed by incubation for 20 minutes at room temperature in complete darkness. Again, absorbance was measured at $412 \mathrm{~nm}$ (background absorption) and $630 \mathrm{~nm}$ (reference). The concentration of free thiol levels was calculated in comparison with the calibration curve. Plasma free thiol concentrations were corrected for plasma albumin by dividing free thiols trough albumin concentrations, since albumin is the most abundant human plasma protein, and is the predominant source of thiols (18).

\section{Measurement of calprotectin}

The serum calprotectin levels were quantified using MRP8/14 ELISA kit (BÜHLMANN, Schönenbuch, Switzerland) using the DS2 ELISA robot (DS2, Dynex, Chantilly, USA) according to manufacturer's instructions to determine the neutrophil activation. Inter assay coefficients of variation (CV) were $12 \%$ and $6.8 \%$ at levels of 1.47 and $5.81 \mathrm{ug} / \mathrm{ml}$, respectively.

\section{Measurement of plasma NGAL}

NGAL was measured in routinely collected lithium heparin plasma samples using the BioPorto NGAL Test (Bioporto Diagnostics, Hellerup, Denmark) in the Department of Laboratory Medicine on a Roche Modular 
P800 chemistry platform (Roche, Mannheim, Germany). According to the manufacturer, the NGAL test is validated for NGAL levels between 25 and $5000 \mathrm{mg} / \mathrm{L}$. Overall, the CV is $2.9 \%$ at a level of $206 \mathrm{mg} / \mathrm{L}$ and $2.3 \%$ at a level of $511 \mathrm{mg} / \mathrm{L}$.

\section{Statistical analysis}

Data analysis and data visualization were performed using R studio (RStudio Team (2015). RStudio: Integrated Development for R. RStudio, Inc., Boston, USA). Descriptive statistics were presented as means \pm standard deviations (SD), medians [interquartile ranges, IQR] (in case of skewed distributions), and proportions $n$ with corresponding percentages $(n, \%)$. Comparisons between groups for continuous variables were performed using independent sample $t$-tests, Mann-Whitney Utests, one-way analysis of variance (ANOVA) or Kruskall-Wallis tests, while for nominal variables chi-square tests or Fisher's exact tests were performed, as appropriate. The normality testing was performed using Q-Q plots. Correlations were tested by either Pearson's correlation coefficients $(r)$ or Spearman's rank correlation coefficients $(\rho)$, depending on normality of distribution, respectively. Associations between covariates and levels of thiols and renal function were calculated using univariable linear regression analysis, followed by multivariable linear regression analysis of selected covariates (univariate $F<0.2$ ). Finally, a logistic regression analysis (odds ratios with $95 \%$ confidence intervals [CI]) was performed to study associations between covariates and the presence of AKI. Statistical significance was defined as a two-tailed $P$-value $\leq 0.05$.

\section{Results}

\section{Baseline characteristics of the study population}

Initially, a total of 361 subjects consented to participate in the study. However, 60 patients were excluded because limited plasma samples were available. Therefore, data from 301 patients were included for analysis, of which 111 patients were female (37\%; Table 1). The median age of the patients was 63 [IQR 54-71] years. In total, 43 (14\%) patients had AKI upon ICU admission with higher median levels of both baseline serum creatinine (90 [IQR 70-106] $\mu \mathrm{mol} / \mathrm{L}$ ) and median creatinine levels upon ICU admission (168 [IQR 139-244] $\mu \mathrm{mol} / \mathrm{L})$ compared to 258 (85.7\%) patients without AKI upon admission (79 [68-94] $\mu \mathrm{mol} / \mathrm{L} ; p<0.05$ and 69 [58-84] $\mu \mathrm{mol} / \mathrm{L} ; p<0.05 ;$ Table 1). Chronic kidney disease (CKD) and diabetes mellitus (DM) were more commonly observed in patients with AKI upon admission $(p<0.05)$ compared to non-AKI patients. Of all patients, $53(18 \%)$ were admitted with a confirmed infection, of which $43(19 \%)$ patients were admitted with the diagnosis of sepsis. Of all patients with $\mathrm{AKI}, 42 \%$ had an infection, of which $98 \%$ was eventually classified as sepsis. $32.6 \%$ of the patients with AKI had scheduled and unscheduled post-operative admission while $72 \%$ of the patients without AKI had a post-operative admission $(p<0.01)$. Patients with AKI upon admission had higher plasma levels of $\mathrm{C}$-reactive protein (CRP) and higher urinary albumin excretion $(p<0.001)$ at ICU admission. Sixteen patients $(4.9 \%)$ died within 7 days and 34 (10.5\%) within 28 days of follow-up; the mortality rate at day 7 and 28 was higher in patients with AKI upon admission as compared to patients without AKI upon admission $(p<0.05)$. 
Table 1

Baseline characteristics

\begin{tabular}{|c|c|c|c|c|}
\hline Characteristics & Total $(n=301)$ & $\begin{array}{l}\text { No AKI at admission }(n= \\
258[85.7 \%])\end{array}$ & $\begin{array}{l}\text { AKI at admission }(n= \\
43[14.3 \%])\end{array}$ & $\begin{array}{l}p- \\
\text { value }\end{array}$ \\
\hline Age (years) & $63[54,71]$ & $62[53,70]$ & $66[55,74]$ & 0.064 \\
\hline Gender (\% female) & $111(36.9)$ & $97(37.6)$ & $14(32.6)$ & 0.643 \\
\hline $\mathrm{BMI}\left(\mathrm{kg} / \mathrm{m}^{2}\right)$ & $\begin{array}{l}22.7[20.5,25, \\
1]\end{array}$ & $22.7[20.7,25.0]$ & $22.3[19.4,25.6]$ & 0.704 \\
\hline $\begin{array}{l}\text { Baseline creatinine } \\
(\mu \mathrm{mol} / \mathrm{L})\end{array}$ & $79[68,95]$ & $79[68,94]$ & $90[70,106]$ & 0.013 \\
\hline \multicolumn{5}{|l|}{ Comorbidities } \\
\hline CKD (\%) & $15(5.0)$ & $5(1.9)$ & $10(23.3)$ & $\begin{array}{l}<.001 \\
0.01\end{array}$ \\
\hline CVD (\%) & $33(11.1)$ & $25(9.8)$ & $8(19.0)$ & 0.131 \\
\hline DM (\%) & $52(17.3)$ & $36(14.0)$ & $16(37.2)$ & $\dot{5} 001$ \\
\hline Malignancy (\%) & $22(6.9)$ & $19(8.6)$ & $3(3.8)$ & 0.255 \\
\hline Operation (\%) & $200(66.4)$ & $186(72.1)$ & $14(32.6)$ & $\begin{array}{l}<.001 \\
0.01\end{array}$ \\
\hline Infection (\%) & $53(17.6)$ & 35 (13.6) & $18(41.9)$ & $\hat{0} .001$ \\
\hline Sepsis (\%) & $43(18.5)$ & $28(14.4)$ & $15(40.5)$ & $\hat{0} .001$ \\
\hline SIRS score & $3[1,3]$ & $2[1,3]$ & $3[2,3]$ & 0.324 \\
\hline SOFA score & $6[3,7]$ & $5[3,7]$ & $8[6,10]$ & $\begin{array}{l}<.001 \\
0.001\end{array}$ \\
\hline $\begin{array}{l}\text { Mechanical } \\
\text { ventilation (\%) }\end{array}$ & $212(71.1)$ & $176(68.8)$ & 36 (85.7) & 0.039 \\
\hline \multicolumn{5}{|l|}{ Vital parameters } \\
\hline Heart rate (bpm) & $80[70,100]$ & $80[70,98]$ & $88[75,100]$ & 0.181 \\
\hline MAP $(\mathrm{mmHg})$ & $\begin{array}{l}\text { 83.7 [72.5, } \\
98.3]\end{array}$ & $85.0[73.3,98.7]$ & $77.5[66.8,93.3]$ & 0.131 \\
\hline
\end{tabular}

Data are presented as median [IQR] or proportions with corresponding percentages (\%). P-values were calculated using a two-tailed Mann Whitney $U$ test or chi-squared test, while significant differences are indicated in bold. CKD, chronic kidney disease; CVD, cardiovascular disease; DM, diabetes mellitus; SIRS, Systemic Inflammatory Response Syndrome; SOFA, sequential organ failure assessment score; MAP, mean arterial pressure; CRP, C-reactive protein; APACHE, Acute Physiology And Chronic Health Evaluation. 


\begin{tabular}{|c|c|c|c|c|}
\hline Characteristics & Total $(n=301)$ & $\begin{array}{l}\text { No AKI at admission }(n= \\
258[85.7 \%])\end{array}$ & $\begin{array}{l}\text { AKI at admission }(n= \\
43[14.3 \%])\end{array}$ & $\begin{array}{l}p- \\
\text { value }\end{array}$ \\
\hline $\begin{array}{l}\text { Respiratory rate } \\
\text { (resp / min) }\end{array}$ & $18[15,25]$ & $16[14,20]$ & $25[18,38]$ & 0.002 \\
\hline $\begin{array}{l}\text { Body temperature } \\
\left({ }^{\circ} \mathrm{C}\right)\end{array}$ & $\begin{array}{l}36.1[35.4 \\
37.2]\end{array}$ & $36.4[35.5,37.3]$ & $35.7[35.1,35.9]$ & 0.078 \\
\hline \multicolumn{5}{|l|}{ Laboratory values } \\
\hline CRP (mg/L) & $\begin{array}{l}10.40[2.80 \\
59.75]\end{array}$ & $9.00[2.40,50.58]$ & $66.10[14.15,117.25]$ & $\begin{array}{l}<.001 \\
0.001\end{array}$ \\
\hline Leucocytes(10^9/L) & $12.6[9.4,16.5]$ & $12.5[9.3,16.3]$ & $12.9[10.8,18.7]$ & 0.274 \\
\hline $\begin{array}{l}\text { Thrombocytes } \\
\left(10^{\wedge} 9 / \mathrm{L}\right)\end{array}$ & $187[142,243]$ & $187[144,242]$ & $167[131,262]$ & 0.898 \\
\hline Bilirubin $(\mu \mathrm{mol} / \mathrm{L})$ & $9[6,15]$ & $9[6,15]$ & $12[7,17]$ & 0.165 \\
\hline Albumin $(\mathrm{g} / \mathrm{L})$ & $30[25,34]$ & $30[26,34]$ & $31[25,33]$ & 0.285 \\
\hline Creatinine $(\mu \mathrm{mol} / \mathrm{L})$ & $73[59,92]$ & $69[58,84]$ & $168[139,244]$ & $\begin{array}{l}<.001 \\
0.00\end{array}$ \\
\hline $\begin{array}{l}\text { Calprotectin } \\
(\mu \mathrm{g} / \mathrm{mL})\end{array}$ & $\begin{array}{l}4.13[2.44 \\
6.75]\end{array}$ & $3.92[2.34,6.60]$ & $4.44[3.12,8.99]$ & 0.108 \\
\hline Thiols ( $\mu \mathrm{mol} / \mathrm{L})$ & $\begin{array}{l}256.24[161.42, \\
374.45]\end{array}$ & $247.57[166.82,393.23]$ & $\begin{array}{l}211.01[90.05, \\
284.31]\end{array}$ & 0.002 \\
\hline APACHE II & $15[11,20]$ & $14[11,18]$ & $22[18,26]$ & $\begin{array}{l}<.001 \\
0.001\end{array}$ \\
\hline APACHE IV & $48[34,68]$ & $44[33,59]$ & $83[66,102]$ & $\begin{array}{l}< \\
0.001\end{array}$ \\
\hline $\begin{array}{l}<7 \text { day Mortality } \\
(\%)\end{array}$ & $18(6.0)$ & $11(4.3)$ & $7(16.3)$ & 0.006 \\
\hline $\begin{array}{l}<28 \text { day Mortality } \\
(\%)\end{array}$ & $32(10.6)$ & $20(7.8)$ & $12(27.9)$ & $\langle .001$ \\
\hline \multicolumn{5}{|c|}{$\begin{array}{l}\text { Data are presented as median [IQR] or proportions with corresponding percentages (\%). P-values were } \\
\text { calculated using a two-tailed Mann Whitney U test or chi-squared test, while significant differences } \\
\text { are indicated in bold. CKD, chronic kidney disease; CVD, cardiovascular disease; DM, diabetes mellitus; } \\
\text { SIRS, Systemic Inflammatory Response Syndrome; SOFA, sequential organ failure assessment score; } \\
\text { MAP, mean arterial pressure; CRP, C-reactive protein; APACHE, Acute Physiology And Chronic Health } \\
\text { Evaluation. }\end{array}$} \\
\hline
\end{tabular}

Reduced plasma free thiol levels were associated with AKI upon admission.

Patients admitted to the ICU with AKI had significantly lower unadjusted plasma free thiol levels (211.0 [90.8-284.3] $\mu \mathrm{mol} / \mathrm{L})$ as compared to patients without AKI upon admission (247.6 [166.8-393.2] $\mu \mathrm{mol} / \mathrm{L}$; 
$p<0.01$; Fig. 1, A). Similarly, after adjustment to albumin levels, patients with AKI upon admission had lower albumin-adjusted plasma free thiols levels $(7.3[3.5-9.0] \mu \mathrm{mol} / \mathrm{g}$ ) compared to critically ill patients without AKI (8.5 [5.8-11.3] $\mu \mathrm{mol} / \mathrm{g} ; p<0.05 ; \mathrm{Fig} .1$, B). Albumin-adjusted plasma free thiol levels inversely correlated with the change in serum creatinine from baseline upon admission to the ICU $(\mathrm{R}=-0.20, p<$ 0.001 ; Fig. $1, C$ ). Reduced albumin-adjusted plasma free thiol levels (OR (odds ratio) $=0.87$ ), increased APACHE IV $(\mathrm{OR}=1.03)$ and diabetes mellitus $(\mathrm{OR}=2.68)$ were independently associated with an increased risk of AKI (Table 2). Admission to the ICU other than scheduled or unscheduled surgery, was associated with a decreased risk of AKI (OR $=0.34$; Table 2). Gender, age, CRP and sepsis were not associated with $\mathrm{AKI}$ in the multivariable logistic regression model.

Table 2

Plasma thiols are associated with AKI in multivariable logistic regression model.

\begin{tabular}{|lllll|}
\hline & Univariate & & Multivariate & \\
\cline { 2 - 5 } & Odds ratio (95\% Cl) & p-value & Odds ratio (95\% Cl) & p-value \\
\hline Constant & & $--3.848(-5.283--2.577)$ & $<0.001$ \\
\hline Plasma thiols $(\mu \mathrm{mol} / \mathrm{g})$ & $-0.119(-0.208--0.035)$ & 0.007 & $-0.106(-0.246--0.005)$ & 0.047 \\
\hline APACHE IV & $0.043(0.030-0.056)$ & $<0.001$ & $0.039(0.026-0.054)$ & $<0.001$ \\
\hline Diabetes mellitus & $1.056(0.352-1.735)$ & 0.003 & $1.032(0.122-1.924)$ & 0.024 \\
\hline Admission via OR & $-1.649(-2.327-1.006)$ & $<0.001$ & & \\
\hline CRP (mg/L) & $0.006(.003-.010)$ & $<0.001$ & & \\
\hline $\begin{array}{l}\text { Sepsis } \\
\text { Plasma thiol levels, APACHE and diabetes mellitus score were associated with AKI. Other factors that } \\
\text { entered the model were admission via OR, operation room; CRP, C-reactive protein and sepsis. Plasma } \\
\text { thiol levels were adjusted for serum albumin. Model characteristics: Chi-square }=77.003, \mathrm{df}=4, \mathrm{~N}=\end{array}$ \\
\hline
\end{tabular}

\section{Association of plasma free thiol levels with the course of AKI during ICU admission}

To assess whether plasma free thiol levels can predict the course of AKI, we correlated the plasma free thiol level measured upon admission with the serum creatinine level that was measured daily during admission. Median serum creatinine levels upon admission were higher in patients with AKI progression (91 [72-131] $\mu \mathrm{mol} / \mathrm{L})$ as compared to patients without AKI progression $(72$ [59-88] $\mu \mathrm{mol} / \mathrm{L} ; p<0.05$; Supplementary Table S2; Appendix). In both AKI progression and new-onset AKI, no significant differences were found in albumin-adjusted plasma free thiol levels ( $p>0.05$; Fig. 1, D-E). Albuminadjusted plasma free thiol levels were significantly reduced in patients with sepsis (8.30 [5.52-10.64] $\mu \mathrm{mol} / \mathrm{g}$ ) compared to patients without sepsis (6.95 [3.72-8.92] $\mu \mathrm{mol} / \mathrm{g} ; p<0.05 ;$ Fig. 2, A). When 
separating AKI patients into groups with and without sepsis, we observed that patients with sepsisassociated AKI had lower levels of albumin-adjusted free thiol levels $(6.8[2.0,7.9] \mu \mathrm{mol} / \mathrm{g}) \mathrm{compared}$ to patients without both, sepsis and AKI $(8.4[5.8,10.8] \mu \mathrm{mol} / \mathrm{g} ; p<0.05)$. However, we found no difference in plasma albumin-adjusted free thiol levels in patients with AKI without sepsis $(7.0[3.5 ; 9.5] \mu \mathrm{mol} / \mathrm{g})$ compared to patients without AKI and/or sepsis ( $p>0.05$; Fig. $2, \mathrm{~B})$. Together, these data suggest that free thiol levels are mainly reduced in sepsis-associated AKI.

\section{Plasma free thiol levels were associated with age, serum albumin and creatinine levels, and inflammatory parameters}

Higher age $(B=-0.72, p<0.001)$, higher levels of neutrophil gelatinase-associated lipocalin (NGAL) $(B=$ $-0.002, p<0.05)$, creatinine and CRP $(B=-0.01$ and $B=-0.01, p<0.05)$, and lower serum albumin $(B=0.47$, $p<0.001$; Table 3 ) were associated with lower plasma free thiol levels. In contrast, plasma levels of calprotectin did not associate with plasma free thiol levels ( $p>0.05$; Fig. $3, \mathrm{~B})$. Of note, calprotectin and NGAL were positively correlated with each other $(p<0.05$; Fig. $3, C)$. 
Table 3

Age and serum albumin are associated with plasma thiol levels in multivariable linear regression model.

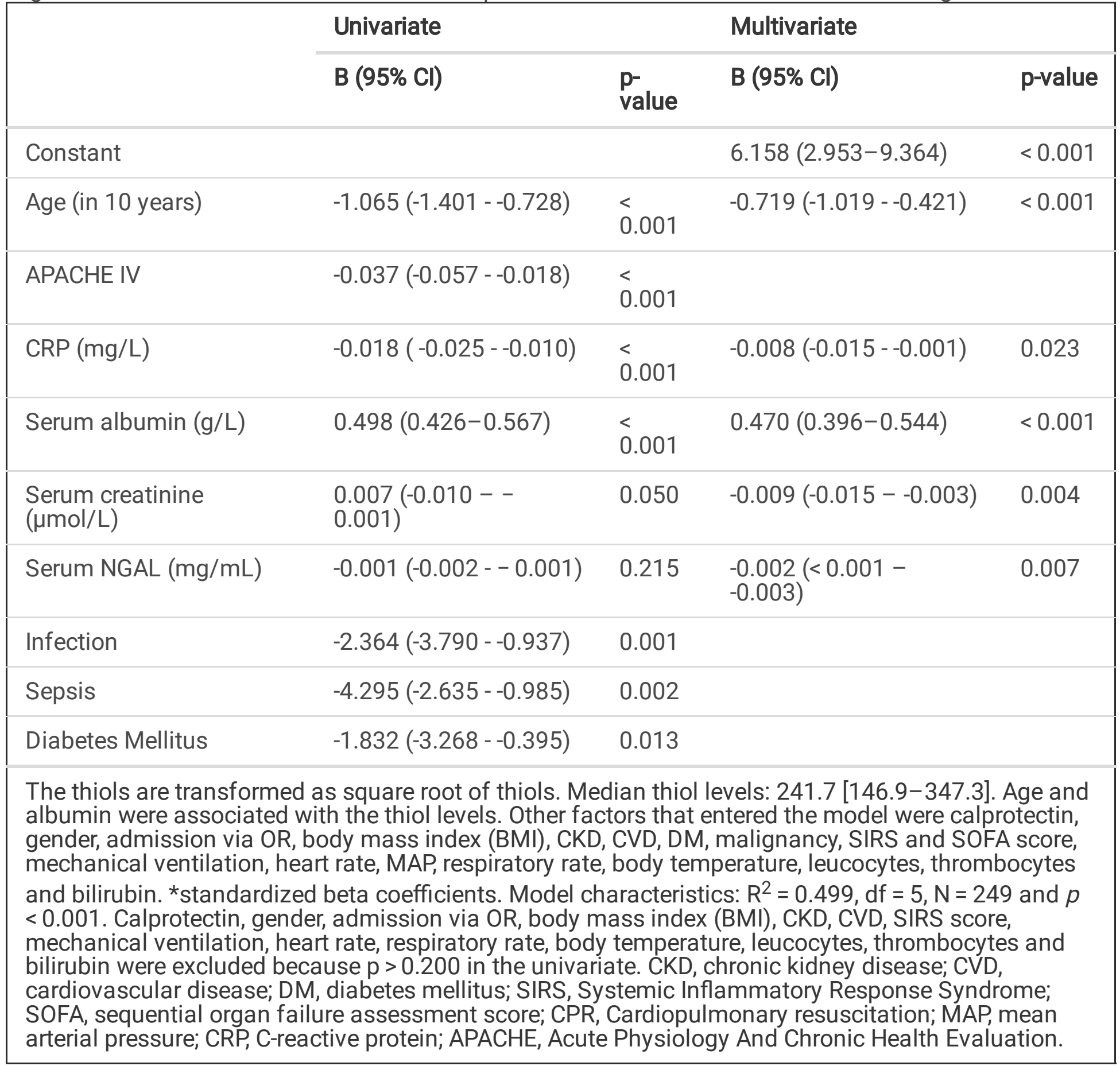

\section{Discussion}

In this study, we investigated the association between albumin-adjusted plasma free thiol levels in relation to (sepsis-associated) AKI in critically ill patients as a biomarker for oxidative stress. Most importantly, we observed that patients admitted to the ICU with AKI had significantly lower levels of plasma free thiols as compared to patients without AKI. However, plasma free thiol levels upon admission were not significantly different between patients with and without new-onset AKI or 
progression of AKI within 48 hours. Furthermore, patients with sepsis had significantly reduced levels of plasma free thiols upon admission compared to patients without sepsis. If we separated the AKI patients into groups with and without sepsis, we found patients with sepsis-associated AKI had lower levels of albumin-adjusted free thiol levels compared to patients without sepsis and AKI. Additionally, we observed that plasma free thiol levels were associated with age, CRP, serum albumin, serum creatinine and serum NGAL. In contrast, however, calprotectin did not correlate with plasma free thiol levels.

Oxidative stress plays an important role in the pathogenesis of AKI in critically ill patients (3). The excessive production of free radicals overpowering the antioxidant machinery results in oxidative stress, which is in turn responsible for extensive cellular and molecular damage. Furthermore, AKI itself is a stimulus for increased oxidative stress, due to mitochondrial dysfunction (3) (24). Over the past decade, multiple biomarkers for AKI have been studied and proposed $(25,26)$. However, no adequate and clinically applicable biomarker for the early prediction of AKI development in critically ill patients is currently available. In clinical practice, creatinine levels are nowadays used as a biomarker to diagnose AKI, but these levels only change once renal failure has occurred (27). In contrast, several other AKI biomarkers have been proposed such as NGAL, IL-18 and urine calprotectin $(25,26)$. NGAL is a biomarker with a high predictive and diagnostic value for AKI which is released during ischemia. However, it is also released during systemic inflammation and therefore lacks specificity $(28,29)$. Given the fact that oxidative stress is a key player in the aetiology of AKI in critical illness, extracellular free thiol levels may be of potential diagnostic and/or predictive value (15). In our study, we demonstrated that patients with AKI upon admission had significantly reduced levels of albumin-adjusted plasma free thiol levels compared to patients without AKI upon admission. In addition, plasma free thiol levels significantly correlated with indicators of renal function, including the change in creatinine upon admission. Previous research demonstrated that patients with hospital-acquired AKI had lower levels of unadjusted plasma free thiols as compared to critically ill patients without AKI and healthy subjects (30). In another study, paediatric patients with AKI also had lower levels of unadjusted plasma free thiols as compared to healthy controls (31).

NGAL and calprotectin are associated with neutrophil activation (11) (28). Calprotectin is a protein heterodimer derived from neutrophils and monocytes which both play a key role during inflammation by inducing activation of other immune cells and enhancing their ROS production, thereby augmenting oxidative stress $(11,32)$. Patients who develop AKI after cardiac surgery had higher levels of plasma calprotectin as compared to patients who did not develop AKI (33). In the present study, we found no correlation between serum calprotectin and plasma free thiol levels. Given the association between calprotectin with inflammation and oxidative stress, the absence of the correlation between calprotectin and free thiols in the present study was against our expectations. This could potentially be explained by the fact that neutrophil activation is of lesser importance in the case of AKI-associated oxidative stress. NGAL is another biomarker of systemic inflammation that is released from neutrophil granules. Both, NGAL and calprotectin, were correlated with each other. However, NGAL is also rapidly induced and released from injured kidney tissue, and is therefore a less specific biomarker of neutrophil activation (28) $(3,25)$. Further, it is an important contributor to free radical generation (3). As described earlier, higher 
levels of NGAL are associated with AKI $(25,26)$. Our results showed higher plasma levels of NGAL in patients with sepsis-associated AKI compared to controls. Further, in our study, plasma levels of NGAL correlated with plasma free thiols. Together, plasma free thiols are associated with NGAL, but apparently not with calprotectin levels. This may implicate that a decrease in plasma free thiol levels would be more associated with a different source of free radicals than neutrophil activation.

Since plasma free thiols were decreased in AKI, it might also identify patients at risk for the development of AKI. However, we did not detect a significant difference in albumin-adjusted plasma free thiol levels in patients that developed AKI compared to patients who did not develop AKI during their ICU stay.

Furthermore, albumin-adjusted plasma free thiol levels showed no significant difference between patients with AKI progression and without AKI progression during ICU admission. Based on these findings, it may be questioned if albumin-adjusted plasma free thiol levels could be used as an early indicator of AKI development as was originally hypothesized. This suggests that AKI itself is associated with a decrease in free thiols.

Strengths of this study comprise the large and extensively characterized study population consisting of patients who were admitted to the ICU in our university medical centre. There was no selection bias, since all patients admitted to the ICU as subsequent admissions were included between January 2014 and April 2014. All relevant demographic, clinical and biochemical information was available for each patient from admission upon to 8 days. Until date, to our best knowledge, no other study focused on unraveling the relationship between (albumin-adjusted) plasma free thiols and AKI. However, our study also has several limitations that have to be considered. For instance, we did not have plasma samples available from every patient in our cohort, which downsized our sample size for the plasma free thiol levels analyses to $83,4 \%$ of included patients. Further, most patients stayed for a short amount of time at the ICU, therefore we lost patients after day 1 . Since our study was performed in a tertiary academic care centre, patients enrolled in our study more frequently had complicated pathologies including complex oncological disease and post-transplantation complications. This may limit the generalizability of our results to other studies on critically ill patients.

\section{Conclusion}

In this study, albumin-adjusted plasma free thiol levels were significantly reduced in patients with AKI upon admission as compared to patients without AKI. However, reduced plasma free thiol levels were not associated with the progression and course of AKI in a large cohort of critically ill patients. As thiols are central components of the extracellular antioxidant network and key transducing elements in redox signalling, lower free thiol levels are reliably indicative of increased levels of oxidative stress. Here, we demonstrated that plasma free thiol levels are associated with AKI and may become an useful pathophysiological indicator of AKI.

\section{Declarations}




\section{Ethics approval and consent to participate}

The Medical Ethics Review Committee (in Dutch: 'Medische Ethische Toetsingscommissie', (METc)) of the UMCG reviewed and waived this study (METC 2013/174).

\section{Consent for publication}

Not applicable.

\section{Availability of data and materials}

The datasets used and/or analysed during the current study are available from the corresponding author on reasonable request.

\section{Competing interests}

The authors declare that they have no competing interests.

\section{Funding}

No funding was received for this study.

\section{Authors' contributions}

Responsible for the conceptualized: HRB, MM and HG; data and sample collection on the ICU: JK and JGZ; measurement of thiols: MLCB and ECS; measurement of calprotectin:

ACMK; analysis and interpretation of the data: LB, HRB and ARB; Drafting of the manuscript: $L B$ and HRB; critical revision of the manuscript: JM, JK, JGZ, MM, HG, ARB and ECS. All authors read and approved the final manuscript.

\section{Acknowledgements}

We want to thank everyone who contributed to the article.

\section{References}

1. Ronco C, Bellomo R, Kellum JA. Acute kidney injury. Lancet. 2019 Nov 23;394(10212):1949-64; doi: 10.1016/S0140-6736(19)32563-2

2. Kidney Disease: Improving Global Outcomes (KDIGO) Acute Kidney Injury Work Group KDIGO Clinical Practice Guideline for Acute Kidney Injury. Kidney Int Suppl. 2:1-138, 2012.

3. Pavlakou P, Liakopoulos V, Eleftheriadis T, Mitsis M, Dounousi E. Oxidative Stress and Acute Kidney Injury in Critical Illness: Pathophysiologic Mechanisms - Biomarkers - Interventions, and Future 
Perspectives. Oxid Med Cell Longev. 2017;2017:6193694; doi: 10.1155/2017/6193694.

4. Case J, Khan S, Khalid R, Khan A. Epidemiology of acute kidney injury in the intensive care unit. Crit Care Res Pract. 2013;2013:479730; doi: 10.1155/2013/479730.

5. Rudd KE, Johnson SC, Agesa KM, Shackelford KA, Tsoi D, Kievlan DR, et al. Global, regional, and national sepsis incidence and mortality, 1990-2017: analysis for the Global Burden of Disease Study. Lancet. 2020 Jan 18;395(10219):200-11; doi: 10.1016/S0140-6736(19)32989-7.

6. Singer M, Deutschman CS, Seymour C, Shankar-Hari M, Annane D, Bauer M, et al. The third international consensus definitions for sepsis and septic shock (sepsis-3). Vol. 315, JAMA. 2016 Feb 23;315(8):801-10. doi: 10.1001/jama.2016.0287.

7. Bouglé A, Duranteau J. Pathophysiology of Sepsis-Induced Acute Kidney Injury: The Role of Global Renal Blood Flow and Renal Vascular Resistance. In: Contributions to Nephrology. Karger Publishers. 2011;174:89-97. doi: 10.1159/000329243.

8. Wan L Bagshaw SM Langenberg C Saotome T May C Bellomo R: Pathophysiology of septic acute kidney injury: what do we really know?. Crit Care Med 2008; 36: S198-S203.

9. Gomez H, Ince C, De Backer D, Pickkers P, Payen D, Hotchkiss J, et al. A unified theory of sepsisinduced acute kidney injury: Inflammation, microcirculatory dysfunction, bioenergetics, and the tubular cell adaptation to injury. Shock. 2014 Jan;41(1):3-11. doi: 10.1097/SHK.0000000000000052.

10. Lushchak VI. Free radicals, reactive oxygen species, oxidative stress and its classification. Chem Biol Interact. 2014 Dec 5;224:164-75. doi: 10.1016/j.cbi.2014.10.016

11. Kerkhoff C, Nacken W, Benedyk M, Dagher MC, Sopalla C, Doussiere J. The arachidonic acid-binding protein S100A8/A9 promotes NADPH oxidase activation by interaction with p67phox and Rac-2. FASEB J. 2005 Mar;19(3):467-9. doi: 10.1096/fj.04-2377fje.

12. Exline MC, Crouser ED. Mitochondrial mechanisms of sepsis-induced organ failure. Front Biosci 2008 May 1;13:5030-41.

13. Abilés J, de la Cruz AP, Castaño J, Rodríguez-Elvira M, Aguayo E, Moreno-Torres R, et al. Oxidative stress is increased in critically ill patients according to antioxidant vitamins intake, independent of severity: A cohort study. Crit Care. 2006;10(5):R146. doi: 10.1186/cc5068.

14. Marik PE, Khangoora V, Rivera R, Hooper MH, Catravas J. Hydrocortisone, Vitamin C, and Thiamine for the Treatment of Severe Sepsis and Septic Shock: A Retrospective Before-After Study. Chest. 2019 Apr 9;8(4):478. doi: 10.3390/jcm8040478.

15. Tiruppathi C, Finnegan A, Malik AB. Isolation and characterization of a cell surface albumin-binding protein from vascular endothelial cells. Proc Natl Acad Sci U S A. 1996 Jan 9;93(1):250-4. doi: 10.1073/pnas.93.1.250.

16. Balcerczyk A, Grzelak A, Janaszewska A, Jakubowski W, Koziol S, Marszalek M, et al. Thiols as major determinants of the total antioxidant capacity. BioFactors. 2003;17(1-4):75-82. doi: 10.1002/biof.5520170108.

17. Banne AF, Amiri A, Pero RW. Reduced Level of Serum Thiols in Patients with a Diagnosis of Active Disease. J Anti Aging Med. 2003;6(4):327-34. doi: 10.1089/109454503323028920. 
18. Turell L, Radi R, Alvarez B. The thiol pool in human plasma: The central contribution of albumin to redox processes. Free Radic Biol Med. 2013 Dec;65:244-253. doi:

10.1016/j.freeradbiomed.2013.05.050.

19. Winterbourn CC, Kettle AJ, Hampton MB. Reactive Oxygen Species and Neutrophil Function. Annu Rev Biochem. 2016 Jun 2;85:765-92. doi: 10.1146/annurev-biochem-060815-014442.

20. Závada J, Hoste E, Cartin-Ceba R, Calzavacca P, Gajic O, Clermont G, et al. A comparison of three methods to estimate baseline creatinine for RIFLE classification. Nephrol Dial Transplant. 2010 Dec;25(12):3911-8. doi: 10.1093/ndt/gfp766.

21. Vincent JL, Moreno R, Takala J, Willatts S, De Mendonça A, Bruining H, et al. The SOFA (Sepsisrelated Organ Failure Assessment) score to describe organ dysfunction/failure. Intensive Care Med. 1996 Jul;22(7):707-10. doi: 10.1007/BF01709751.

22. Ellman GL. Tissue sulfhydryl groups. Arch Biochem Biophys. 1959 May;82(1):70-7. doi: 10.1016/0003-9861(59)90090-6.

23. Hu ML, Louie S, Cross CE, Motchnik P, Halliwell B. Antioxidant protection against hypochlorous acid in human plasma. J Lab Clin Med. 1993;121(2):257-62.

24. Plotnikov EY, Kazachenko A V., Vyssokikh MY, Vasileva AK, Tcvirkun D V., Isaev NK, et al. The role of mitochondria in oxidative and nitrosative stress during ischemia/reperfusion in the rat kidney. Kidney Int. 2007 Dec;72(12):1493-502. doi: 10.1038/sj.ki.5002568.

25. Schrezenmeier E V., Barasch J, Budde K, Westhoff T, Schmidt-Ott KM. Biomarkers in acute kidney injury - pathophysiological basis and clinical performance. Acta Physiol. 2017 Mar;219(3):554-572. doi: 10.1111/apha.12764.

26. Kashani K, Cheungpasitporn W, Ronco C. Biomarkers of acute kidney injury: The pathway from discovery to clinical adoption. Vol. 55, Clinical Chemistry and Laboratory Medicine. $2017 \mathrm{Jul}$ 26;55(8):1074-1089. doi: 10.1515/cclm-2016-0973.

27. Uchino S. Creatinine. Curr Opin Crit Care. 2010 Dec;16(6):562-7. doi: 10.1097/MCC.0b013e32833ea7f3.

28. Mårtensson J, Bellomo RR. The rise and fall of NGAL in acute kidney injury. Blood Purif. 2014;37(4):304-10. doi: 10.1159/000364937.

29. Koeze J, Van Der Horst ICC, Keus F, Wiersema R, Dieperink W, Kootstra-Ros JE, et al. Plasma neutrophil gelatinase-associated lipocalin at intensive care unit admission as a predictor of acute kidney injury progression, Clinical Kidney Journal, 2020.

30. Qian J, Fang J, Zhu Q, Ma S, Wang W, Zheng Y, et al. Serum Protein Thiol Levels in Patients with Hospital-Acquired Acute Kidney Injury. Kidney Blood Press Res. 2015;40(6):623-9. doi: 10.1159/000368538.

31. Ayar G, Sahin S, Yazici MU, Neselioglu S, Erel O, Bayrakci US. Effects of Hemodialysis on ThiolDisulphide Homeostasis in Critically III Pediatric Patients with Acute Kidney Injury. Biomed Res Int. 2018 Sep 25;2018:1898671. doi: 10.1155/2018/1898671 
32. Tan X, Zheng X, Huang Z, Lin J, Xie C, Lin Y. Involvement of S100A8/A9-TLR4-NLRP3 Inflammasome Pathway in Contrast-Induced Acute Kidney Injury. Cell Physiol Biochem. 2017;43(1):209-222. doi: $10.1159 / 000480340$.

33. Nikolakopoulou Z, Hector LR, Creagh-Brown BC, Evans TW, Quinlan GJ, Burke-Gaffney A. Plasma S100A8/A9 heterodimer is an early prognostic marker of acute kidney injury associated with cardiac surgery. Biomark Med. 2019 Feb;13(3):205-218. doi: 10.2217/bmm-2018-0238.

\section{Figures}

A
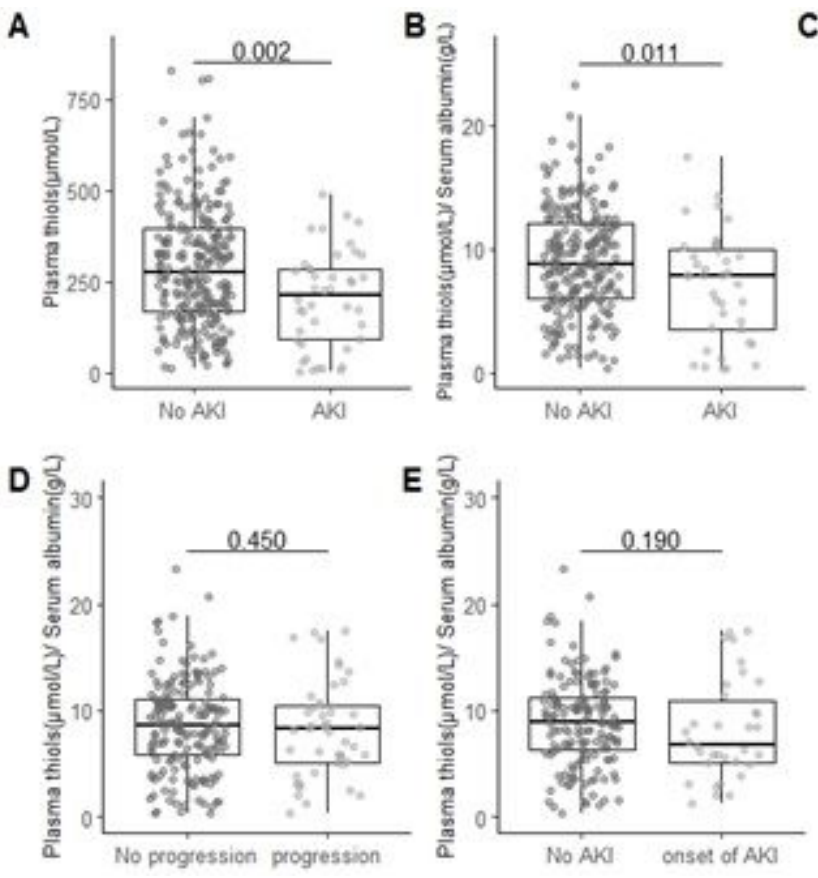

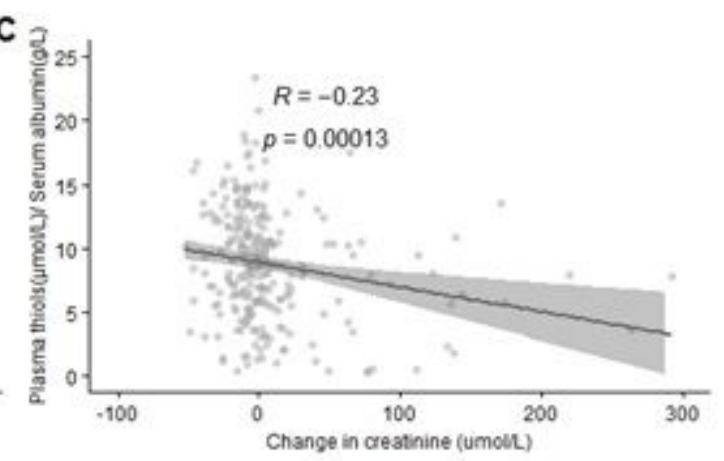

\section{Figure 1}

Acute kidney injury is associated with lowered plasma free thiol levels. A: plasma free thiol levels are lower in patients with AKI as compared to non-AKl; B: after adjusting the plasma free thiol levels for the plasma albumin levels, plasma thiol levels remain lower in patients with AKI as compared to non-AKI patients; C: adjusted plasma free thiol level inversely correlated with the change in serum creatinine from baseline to admission; $\mathrm{D}$ : adjusted plasma free thiol levels for albumin are not different than patients with AKI progression within 48 hours as compared to patients without AKI progression; E: adjusted plasma free thiol levels for albumin are not different than patients with AKI onset within 48 hours as compared to patients without AKI onset or AKl; Group-differences are calculated using a two-tailed MannWhitney U test; correlations are calculated with Spearman. 

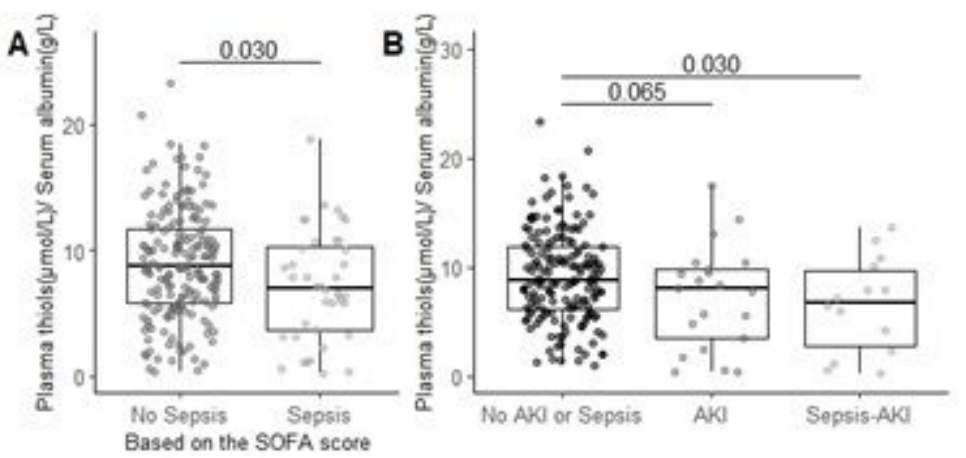

\section{Figure 2}

Patients with sepsis have lower albumin-adjusted free thiol levels in plasma. A: adjusting the plasma free thiol levels for plasma albumin, plasma thiol levels are lower in patients with sepsis as compared to patient without sepsis; B: adjusted plasma free thiol levels are only significant lower in patients with sepsis induced AKI compared to patients without sepsis or AKI. Group-differences are calculated using a two-tailed Mann-Whitney U test.
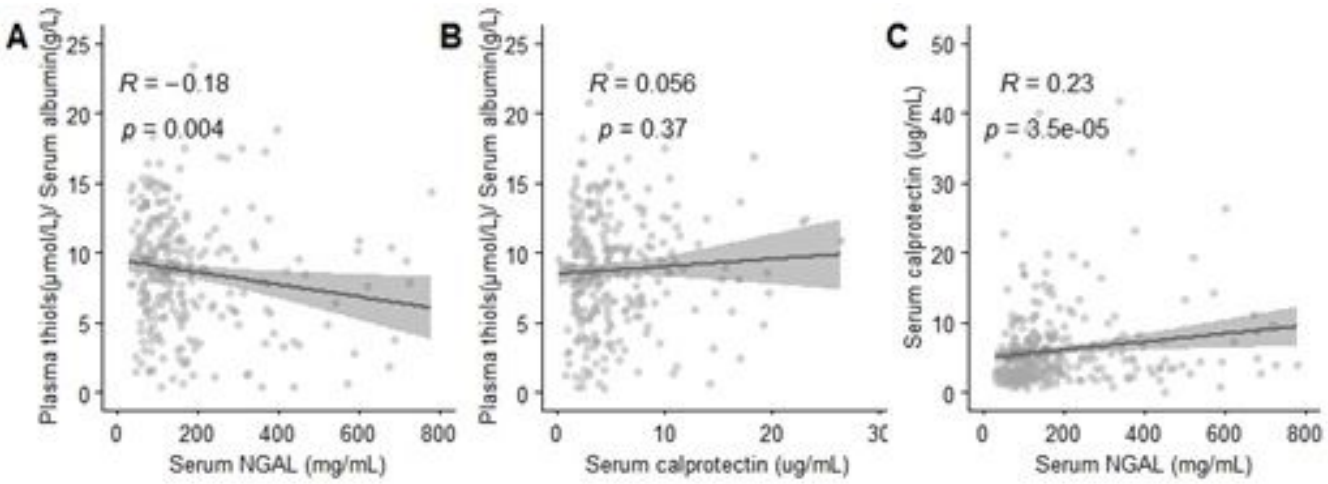

\section{Figure 3}

Albumin-adjusted plasma thiol levels correlated with NGAL. A: plasma free thiol levels correlate with plasma NGAL levels; B: plasma free thiol levels do not correlate with plasma calprotectin levels; C: plasma calprotectin levels correlate with plasma NGAL levels. Correlations are calculated with Spearman.

\section{Supplementary Files}

This is a list of supplementary files associated with this preprint. Click to download.

- ArtikelthiolsAKIAppendix.docx 